\title{
A Cognitive Linguistic Study of the English Preposition 'in'
}

\author{
Raghad Fahmi Aajami \\ Department of English, College of Education for Women \\ University of Baghdad, Baghdad, Iraq \\ E-mail: Raghad.fahmi@coeduw.uobaghdad.edu.iq
}

\section{DOI: https://doi.org/10.36231/ coedw/vol30no3.14}

\section{Received 18/4/2019, Accepted 30/6/2019}

\begin{abstract}
The present study aims at analyzing the polysemy of the English preposition in from the cognitive linguistic (CL) point of view using Evans' and Tyler's approach (2003). The perplexity faced by Iraqi second language learners (L2) due to the multi-usages of this preposition has motivated the researcher to conduct this study. Seventy-six second year university students participated in this experimental study. The data of the pre-test and post-test were analyzed by SPSS statistical editor. The results have shown the following: First, a progress of more than $(0.05 \leq)$ has been detected as far as students' understanding of the multiple usages of the preposition in is concerned. Second, the results of the questionnaire have shown a prominent positive change in the students' attitude toward CL approach. Third, the main source of difficulty has been shown to be attributed to the diversity in the semantics of the preposition in. Fourth, CL as an approach has proven its effectiveness in accurately comprehending the semantics of the English preposition in.
\end{abstract}

Key words: cognitive linguistics, semantics, polysemous meaning, preposition
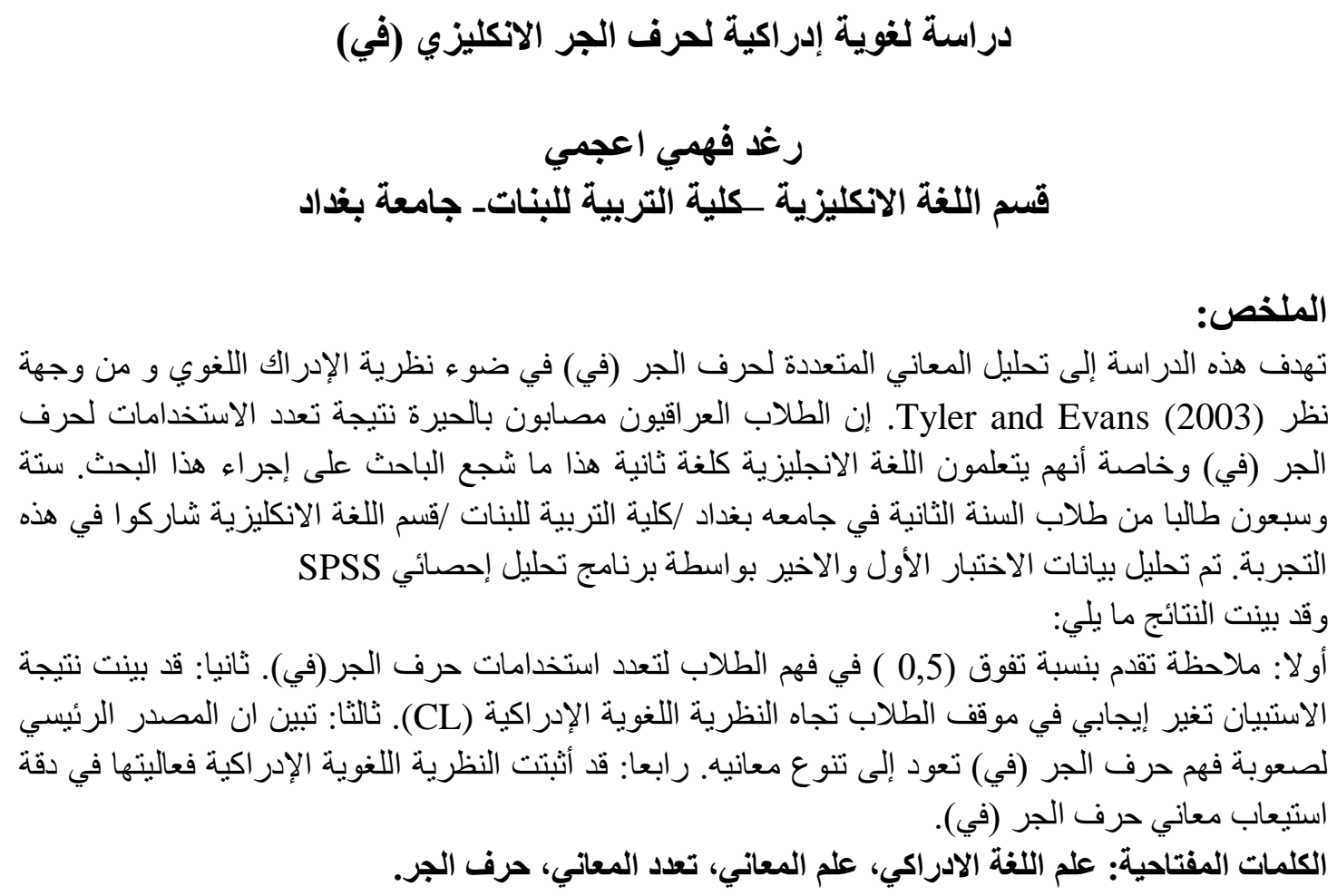


\section{Introduction}

As long as they are L2 learners, Iraqi students face the same difficulties experienced by the other L2 learners in comprehending the polysemy of the English preposition in. This problem has been noticed since (1999) by Celce-Murcia and Larsen-Freeman, "linguists as well as English teachers have long noticed that generally the acquisition of prepositions is a major challenge for L2 learners". In the Arab world, people sometimes use English expressions without prepositions; for example, they say "to discuss" not "to discuss about", or "to marry" not "to marry with" (Saed \& Yassin, 2017). Recognizing the differences between in and other prepositions such on, at, and to needs a deep understanding of these prepositions. For instance, "he sits in/ at the corner" and "we arrive to /in London". Iraqi researchers pay a great attention to the polysemy of English prepositions. Al-Baharani and Al-Robuye (2016) analyzed the semantics of the English preposition at. They explained the problem and offered logical solutions following the CL approach. Aajami (2018a) also identified the problem of the English preposition at, and worked skillfully on two other locative English prepositions, behind and beyond to tackle the polysemy of these prepositions (Aajami, 2018b).

CL approach offers a complete analysis not only to English prepositions, but also to other languages'. It defines the meaning of a preposition in three aspects; clarifies its abstract notion, represents its functional element, and gives the preposition a schematized representation of a spatial configuration between two entities (Tyler \& Evans, 2003). The insights of CL are also adopted in analyzing the English preposition in. Thus, this study is to test the effectiveness of CL approach in accurately and systematically comprehending the English preposition in.

\subsection{Objectives of the Study}

The study aims at examining to which extent CL approach can help increase the participants' ability to elicit the semantics of the English preposition ' $i n$ ' in their speech contexts.

\subsection{Limitations of the Study}

The study was limited to second-year students in the Department of English /College of Education for Women/ University of Baghdad/ Iraq. It was conducted during the academic year 2018/2019. Seventy-six participants were randomly selected by putting their names in a basket, shaking the basket and then randomly selecting the names.

\section{An Overview of Cognitive Linguistics as an Approach}

This section concentrates on CL approach and its useful implementations in defining the semantics of English prepositions for L2 learners. George Lakoff, Ron Langacker, and Len Talmy are considered the founders of CL approach constructed in 1980s. It deals with the language as a tool of organizing, processing, and conveying information (Geeraerts \& Cuyckens, 2010).

Evans (2012) defines CL approach as the study of language in terms of mind, and sociocultural experience which views language as a reflection of general aspects of 
cognition. Language is best studied in the context of use that emerges from it. Evans illustrates the vision of CL approach, saying that meaning and form in the study of language are inseparable. He also clarifies the two main areas of CL concentrations: CL to grammar, which is the study of language organization, and cognitive semantics, which is the study of the conceptual structure of language. Ungerer and Schmid (2001) say that CL approach is "based on our experience of the world and the way we perceive and conceptualize it”.

English prepositions are difficult to be understood since they are polysemous. They are the most repetitive words in English, and have a complex set of uses. Therefore, L2 learners face the challenge of comprehending the English prepositions (CelceMurica \& Larsen-freeman, 1999). Thus, CL approach is used to address this issue for the purpose of analyzing the English prepositions and their semantic networks in terms of spatial scenes, relations, and figurative senses (Mueller, 2016).

The locative English prepositions can create spatial relations among a land mark and an agent. The multiple meanings associated with English prepositions can be represented as being systematically related within a motivated semantic network (Tyler et al., 2011). The central notion of a spatial scene creates conceptualized relations between two entities, spatial experience and interaction. For example "the ball is in the box", the spatial scene in this example means that there is a contact between the ball and the box. This motivates another scene which is described as "there is air in the ball". These relations are important because without the box, the ball will move to another place, and without the ball, the air will not be compressed. The spatial scene involves a support relationship between "the box $\&$ the ball" and "the ball \& the air" (Tyler et al., 2011). The human interaction response to the scene of "the ball is in the box" differs due to the viewer's main concentration. One will concentrate on the relation between the box and ball while another may concentrate on the relation between the ball and the air to check its validity in playing a football game. Thus, one can get the result that there are no identical vantage points. The way a viewer views the physical vantage point of a spatial scene will determine the way that he will interpret it according to his conceptualization (Evans \& Tyler, 2003).

Each central scene can extends different spatial relations in a systematic way. Prepositions that describe a contact relation can develop rotated senses (Boers, 1996). For instance, "the water is in the glass" $\rightarrow$ "the milk is in the glass"; this is called the spatial relations. A figurative sense can also be developed from a spatial scene. Beside the fact that "the water is in the glass" represents a spatial configuration of entities, it further connotes the metaphorical sense that the first entity (the water) is inside and the second entity (the glass) is outside (Boers \& Demecheleer, 1998).

There are many studies that examine the semantics of the English preposition in. Fernando (2000), for instance, finds that "the standard semantic description of the English preposition in has been traditionally understood as a matter of geometric 
configuration of the participants in the spatial relation. The landmark is conceived of as an area or volume, or as a three-dimensional entity, the topological relation of an inclusion being emphasized. The landmark may also be understood as a MEDIUM configuration, in opposition to the geometric conception based upon the idea of a container. Other authors pose a meaning based on the relative function of the participants, i.e. the control of the container over the contained entity. Fernando concentrates on the central meaning from which other senses can be derived. He finds that the English preposition in has many senses: as inclusion as in: 'A second revolution in Rome'; as a definite inclusion, where this definition is given by certain landmarks that are a part of the whole area as in: 'there is a big black point in the bottom of the paper'. Another sense is that of a medium or a surrounding substance as in: 'the jet is in the sky'. The sense of a massive material in which an entity is entirely or partly located in a massive chunk of material like 'a nail is in a wall". The sense of material in which entities are made is spoken of as if included in a medium filled with that material, as in: 'a statute in wood' (Navarro, 2000).

Brala (2008) finds that in is a preposition that has most extensively been dealt with in the literature. On the basis of its treatment in the relevant literature, 'in' seems to have quite a clear 'prototypical' or 'ideal' meaning, and is frequently referred to as 'a basic topological preposition'. The preposition in ( just like its counterparts in other languages under examination) is one of the most frequently used prepositions. It falls in the middle range of a semantic complexity. It is the first preposition to be acquired by children (and, cross-linguistically speaking, the same is true of other words in other languages expressing the concepts of 'containment' and 'support').

Suzanne (2017) descriptively studies the difficulties of using English prepositions by 12 learners. She finds that the English preposition in has a close meaning to other prepositions as on and at especially in terms of time and place. Thus, the Indonesian L2 learners always make mistakes when using the English preposition in. Her study focuses only on showing the errors in using prepositions. There is not any solution to solve the problem of preposition for L2 learners; however, the importance of Suzanne's study is to assure that L2 learners have a real problem in using English prepositions.

Khan and Ali (2017) make a deep investigation about the counterpart of the English preposition in in the Arabic language which is $f i$. They find that the preposition $f i$ or in in English has been used to show the position of an object within the bounded space in physical surroundings. Thus, it carries the locative semantic arguments in each case, and, therefore, in traditional linguistic categorization of prepositions, it has been termed as a preposition of location. They also insist on the necessity of investigating the preposition in light of the immediate communicative context to arrive at a definite conclusion about the meaning of the preposition. Time has been mapped as bounded space through the preposition $f i$. It reveals the sensorimotor neural structures in the 
human conceptual system that have psychological reality in defining the abstract concept of time as a bounded space and that the preposition $f i$ is generated by these structures in the human conceptual system. These structures are also at work in the use of $f i$ in defining other abstract concepts and states through the container image schema. They also find that the preposition $f i$ is also used with nouns relating to discourse as a preposition. In the discourse, too, the preposition attains the spatial meaning of containment. It locates the words on the pages of a book as if they were things in a bounded space or in a container.

Similar to Khan and Ali, a study is conducted to investigate $f i$, the counterpart of English preposition in. This study concentrates on using this preposition in Taizzi dialect in Yemen. They find that ' $f i$ ' has shown a schematic meaning of "containment" as a central meaning from which other senses are emerging. They also find that the preposition $f i$ is used to denote spatial relations where the trajector (TR) is situated on the landmark (LM), the TR is under the LM, the TR is inevitably directed towards the LM, the TR and the LM are facing one another, and others. However, the analysis presented in this paper has established that in all these spatial relations, speakers of the TD cognitively characterized LMs as containers that contain TRs (Mohammed et al., 2012).

\section{Cognitive Semantic Analysis of the Preposition "in"}

This section concentrates on the semantic analysis of the preposition in according to CL approach that is developed by Tyler and Evans (2003). Cognitive semantic analysis can help overcome the perplexity encountered due to the polysemous nature of the preposition in and get accurate comprehension of its usages and expressions. A spatial scene can be viewed physically depending on the vantage point of the viewer. As long as this physical view depends on the viewer, there is no two identical vantage points (Tyler \& Evans, 2004). The first central spatial sense of in can be represented in "the ball is in the box", where box is a land mark. The preposition in refers to a specific place which is a box. It defines the place of the ball. The trajector "the ball" is inside the land mark, "the box". This scene can be represented in the following diagram that is set by the researcher of the present work.

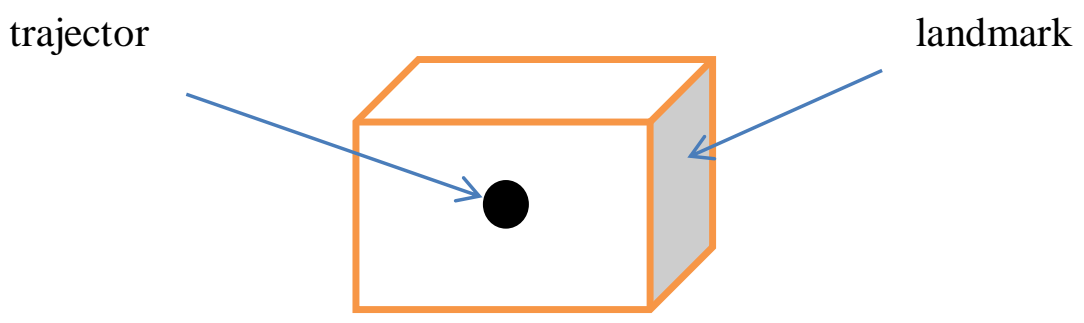

Figure1. The ball is in the box. 
Figure 1 is an example of the spatial relations that can be extended to other examples, as in 'the people are in the car'. In this sentence, in refers to two entities, 'the people' and 'the car'. As in figure 1, in gives the meaning of containment. It can give two main functions: The first one is the meaning of containment, and the second function defines the place of the trajector for the landmark. It can also give a kind of protection for the trajectors in both sentences. Without the box, the ball can move to other places. It is the same for people who may feel hot or cold without the car. Thus, the preposition in gives a central meaning and develops a figurative sense of protection.

Another central sense of in can be represented in 'the jet is in the sky'; here, in conveys the meaning of part of all. In defines the place in which the jet is flying; it also conveys a vertical axis depending on the position of the jet and the vantage point of the viewer. It clearly shows that the physical vantage points do not offer the same view. In shows general place unlike the preposition at which can indicate the definite a place of an object. Hence, the relation between the jet as a part and the sky as a whole place is general. Such a relation can be represented in the following diagram that is set by the researcher.

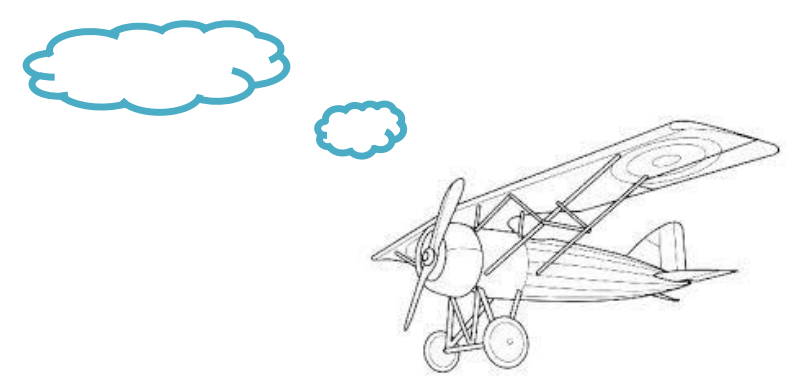

Figure 2. The jet is in the sky.

Source: This figure is set by the researcher.

In figure 2, in represents the position of the part in respect to the whole. It can convey a vertical or horizontal axis according to the medium and the part that is mentioned. For example, 'there is a boat in the sea'; Each example with the preposition in can offer the relation of entities and the places in respect to the viewer. The human being interprets the language and uses it based on his/her understanding to the relations between objects throughout the image schema created as soon as the speaker utters a sentence.

In the above mentioned examples, the analysis of in concentrates only on the one aspect which is place. There is another important aspect that is conveyed by in which is time. In terms of time, in gives a general notion of time. For instance 'My daughter was born in 2018', here, the date of birth is not clear, but it is defined by a general frame which is the year 2018. This scene can be represented in the following diagram that is set by the researcher. 
Source: This figure is set by the researcher

Thus, in can represent a general notion of time that is contained in a specified frame. In another example, 'Tom drinks 4 cups of tea in the morning'; 'morning' here is a period of time that continues from the sunrise to the afternoon. The preposition in connects between two entities, Tom and $\mathbf{4}$ cups of tea and the specified frame of time which is morning. If one compares ' $i n^{\prime}$ ' to the preposition ' $a t^{\prime}$, there is an important difference. It has been learned at school that ' $i n$ ' is used with the morning not 'at', but no one supports a logical piece of evidence for that use. The comparison will be clearly shown in the following diagrams. 'Tom drinks 4 cups of tea in the morning'/ Tom drinks 4 cups of tea at breakfast'. This scene can be represented in the following diagram that is set by the researcher.

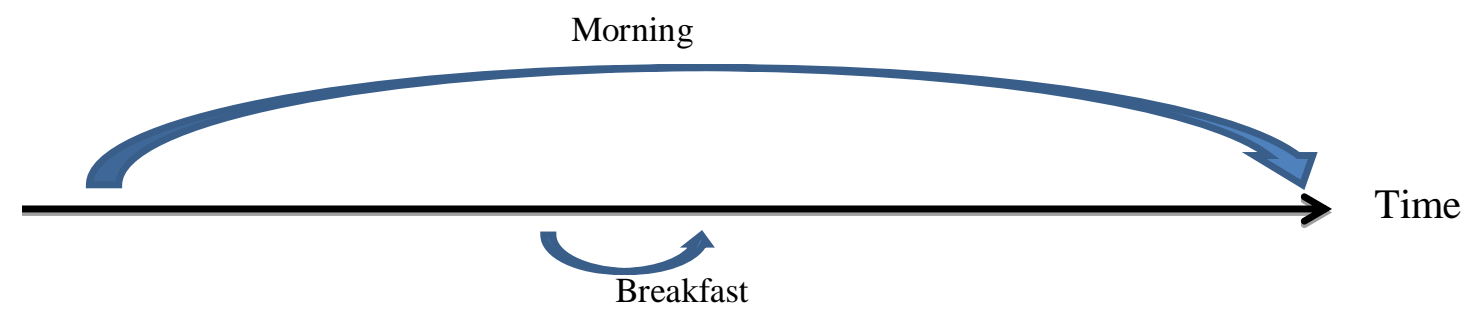

Figure 4. Tom drinks 4 cups of tea in the morning./ Tom drinks 4 cups of tea at breakfast.

Source: This figure is set by the researcher

\section{Experimental Study}

The study aims at enhancing Iraqi students' awareness of CL approach and developing their comprehension ability in interpreting English prepositions. Seventy six students participated in this experiment as an experimental group. The researcher tests the students' information about CL approach in explaining the preposition through testing such items as pictures and drawings. A questionnaire was distributed before and after the experiment to check the change in the students' attitudes and opinions about CL approach. She also arranged an online survey to be filled by the participants before conducting the experiment that discusses the same points of the questionnaire and to offer the participants more flexibility in participating. The researcher prepared an experiment of three points to achieve her goal:

1. Displaying to the students videos and PowerPoint slides that explain the principles of CL approach and its insights: spatial scene, spatial relations, figurative sense, and a metaphorical language;

2. Distributing pictures and asking the students to identify the spatial scenes and relations using the English preposition in; 
3. Asking the students to rephrase sentences with in that contain physical and abstract views in order to develop their metaphorical languages.

The researcher adopts quantitative method in collecting and analyzing the data. SPSS statistical editor; paired sample statistics is used to analyze the students' scores in the pre-test and post-test. Only the result of the analysis of the students' marks is mentioned.

\subsection{Participants and Procedures}

The participants were seventy six second-year students who were mostly of intermediate level in English language. Some of the students have participated in a previous experiment about CL approach. The procedures were done as shown below:

1) The first step: the students were introduced to a detailed introduction to CL approach, and they were further presented with its definition, principles and insights;

2) In the second step, they sat to do the pre-test that contained three categories of questions: images to be analyzed, sentences to be rephrased, and gaps to be filled. The pre-test was set in order to check the participants' ability in explaining the meaning and function of the preposition in;

3) In the third step, at the end of the experiment, the participants sat for the post-test which contained the same categories of the pre-test. The experiment continued in order to reveal the change in the participants' awareness as they dived deeper in the experiment; and

4) In the last step, there was a questionnaire of three questions to check any change in the participants' attitudes toward CL approach.

To sum up, the pre-test and post-test are set to check the participants' progress in grasping CL approach whereas the questionnaire is set to check the participants' attitude toward CL approach.

\subsection{Pre-test}

Seventy six marks were collected by the researcher for the initial examination of the students' abilities in interpreting and analyzing the semantics of in. It is clear that the students have a simple ability to analyze the meaning of in depending on the entities that are connected via a relation to this preposition. Their marks also displayed prodigious difficulty in developing the meaning of this preposition metaphorically. As long as the pre-test has been done after the introductory part of the experiment, the result of the pre-test showed that some the students have a simple clue about CL approach and its insights. The others have displayed good interpretations of CL approach since they have similar experiments with the same theory. It is obvious that some students, ie., about $50 \%$, depended on their initial knowledge when rephrasing the sentences or images. Half of the participants, ie., 50\%, failed to differentiate between the spatial and metaphorical senses. Besides, $60 \%$ of the participants had 
right answers regarding the choice between in, on or at to complete the sentences. The achievements in the pre-test motivated the researcher to pay much more attention to the current methodology to be more effective and clearer when clarifying the meaning and usage of CL approach.

\subsection{Post-test}

After the pre-test, the researcher applied the detailed experiment in nine weeks that involved lectures, workshops, games and tests. For each week, there were three meetings of two hours. The sessions were divided into three parts theoretical, practical, and representational. Those nine weeks were enough for the students to be familiar with CL approach. The researcher through the lectures showed videos and explained CL approach using the experiment of Tyler and Evans (2003). She also used schematic diagrams to present the meaning of the preposition in. In the practical part, the students were divided into groups to represent part of the theory practically. They, the students, chose examples, pictures, and drew diagrams to illustrate the usage of ' $i n$ '. The students worked in groups during the workshops, and they analyzed and identified spatial scenes and relations in sentences and images. They also defined the abstract or the physical vantage point in a sentence or an image and represented some images in real scenes. Sentences with 'in' were rephrased by the students. During that time, students had a clear idea about CL approach. Then, they practiced developing the physical view to spatial relations and spatial configurations.

The post-test contains the same categories of the questions of the pre-test. The marks were collected through a rubric set by the researcher. Throughout checking the results of the post-test and in comparison with the pre-test, the researcher has noticed that the students have a remarkable development in the awareness of CL approach. All students, i.e., $100 \%$ of the participants, could recognize if there is a spatial scene or not in the images and sentences; a matter which reflects that their responsiveness to the first step of comprehension is successfully done. Moreover, $91 \%$ of the participants showed important progress in differentiating the metaphorical usage of the prepositions in question. Besides, $98 \%$ chose the right choice of 'in' to complete the sentences correctly by a suitable preposition.

The tables below accurately analyze the marks using SPSS statistical editor. 


\section{T-Test [DataSet0]}

Paired Samples Statistics

\begin{tabular}{|cc|c|c|c|c|}
\hline & & Mean & $\mathrm{N}$ & Std. Deviation & $\begin{array}{c}\text { Std. Error } \\
\text { Mean }\end{array}$ \\
\hline Pair 1 & Pre-test & 13.00 & 76 & 2.444 & .292 \\
& Post-test & 18.47 & 76 & 1.901 & .227 \\
\hline
\end{tabular}

\section{Paired Samples Correlations}

\begin{tabular}{|ll|c|c|c|}
\hline & & $\mathrm{N}$ & Correlation & Sig. \\
\hline Pair 1 & $\begin{array}{c}\text { Pre-test \& } \\
\text { post-test }\end{array}$ & 76 & .231 & .055 \\
\hline
\end{tabular}

Table (1)* shows the difference between the pre-test and post- test

* Table (1):

If the difference between both tests is less than $(0,05)$, then, the study is invalid.

If the difference between both tests is more than $(0,05)$, then, the study is valid.

Table (1)*, SPSS analysis, shows the students' number and the results in both tests, the pre-test and post-test and the difference between them. The number of students is 76 and their average in the pre-test is 13.00 whereas the post-test is 18.47 . The students show a progress of 5.47 marks. Since the difference between the two means of both tests shows a progress of more than $(0,05)^{*}$, then, this study is valid. The results are in agreement with the research of Evans and Tyler's (2003). This means that CL approach can make a remarkable positive change in students' comprehension of English prepositions.

\section{Questionnaire}

The questionnaire is set in order to show a change in students' attitude toward the cognitive linguistic approach. Further, moving from unfamiliarity to cognition, it seems that the change in the questionnaire's results shows that human experience is enriched. Students' mental perception passes through an experiment that turns their observation about how cognitive linguistics offers a new insight of the semantics of English prepositions in. The questionnaire is distributed before and after the experiment. 


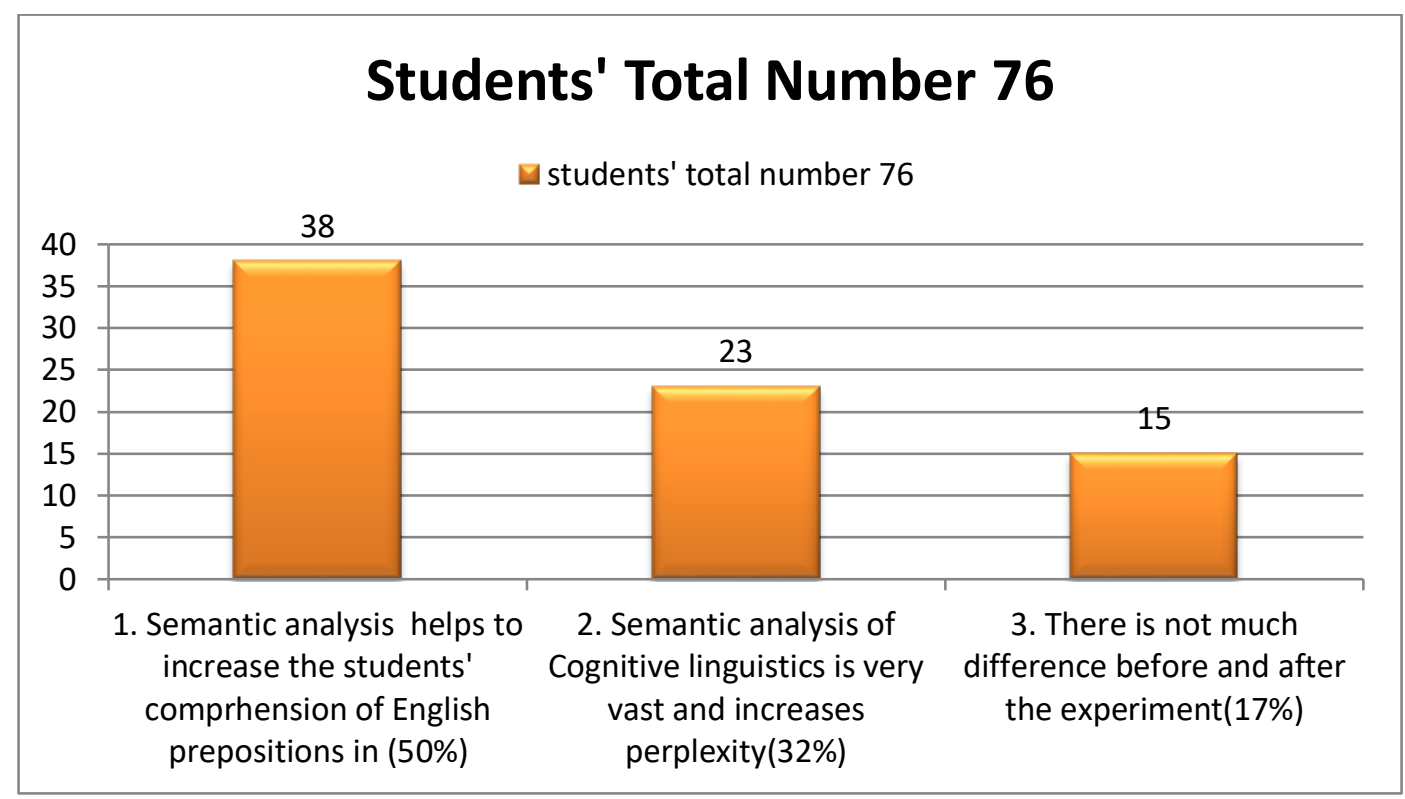

Figure (5): The questionnaire's results before the experiment

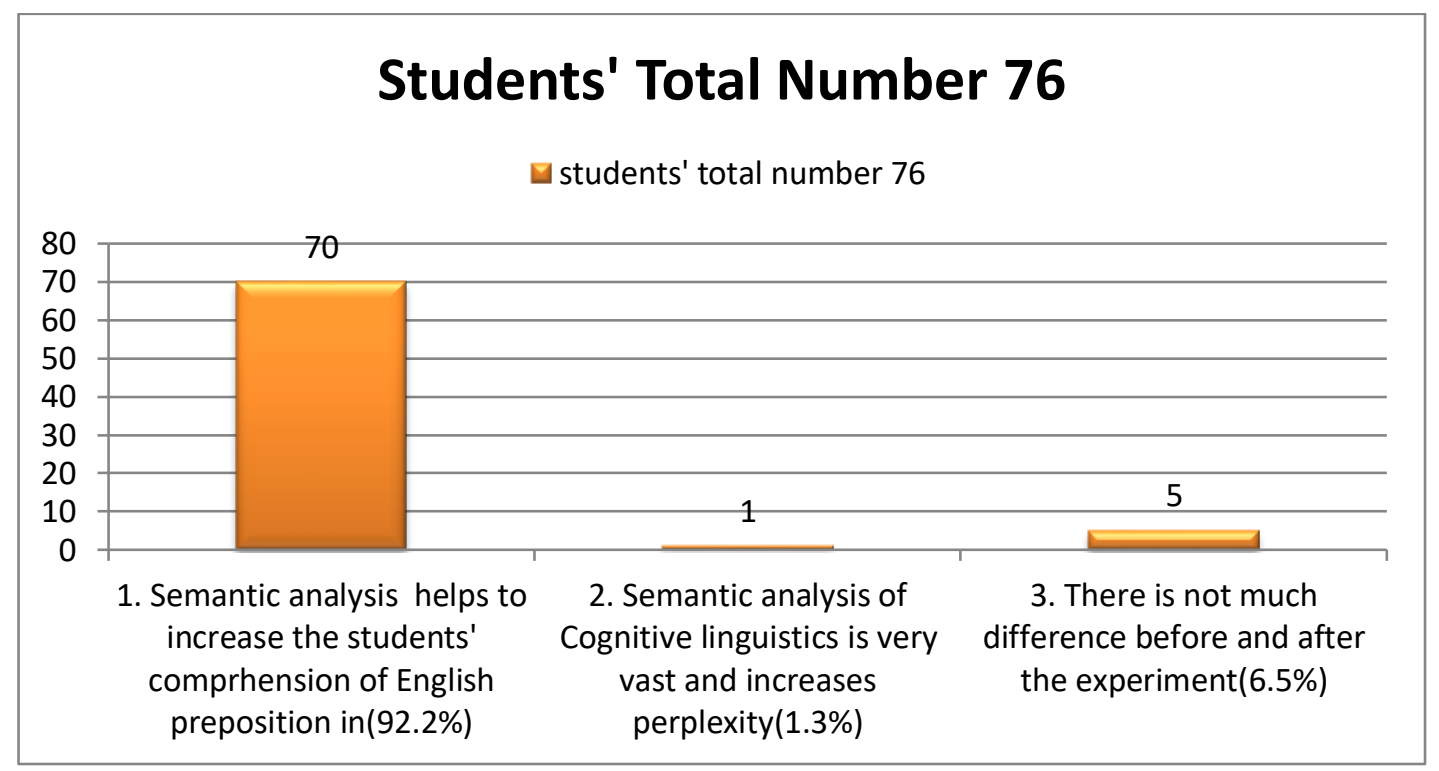

Figure (6): The questionnaire's results after the experiment. 
The questionnaire shows that $92.2 \%$ of the students which represents 70 out of 76 of the students' total number thinks that $\mathrm{CL}$ approach helps bettering up their comprehension of the English preposition ' $i n$ '. Besides, 1.3\%, which represents 1 student out of the total number finds $\mathrm{CL}$ approach difficult and confusing. Moreover, $6.5 \%$, which represents 5 students do not find a big change in their comprehension to the English preposition 'in' by using CL approach. These results of the questionnaire prove a positive change in the students' attitude toward using cognitive linguistics approach to treat English prepositions.

\section{Results and Findings}

This study supports the students' confidence in dealing with the locative English preposition in. It helps them acquire new concepts when analyzing this preposition through their location in the context, and when identifying what it means or implies. As long as they practice explaining the meaning of in using objects from real life, they can also practice what they have learnt in real life.

The results of both tests and the questionnaire evidently show a clear progress in the students' work and attitude toward CL approach.

\section{Conclusion}

This study proves the effectiveness of CL approach in grasping the semantics of English prepositions. According to the results of pre-test and post-test in addition to the results of the questionnaire, it is quite obvious that students have achieved a significant growth in reaching the semantics of the English prepositions in question with a better aspect. This research recommends that paying more concentration for $\mathrm{CL}$ approach is a necessity due to its importance in understanding the interaction between human actions and languages. 


\section{References}

Al-Baharani, R., \& Al-Robuye, M. R. (2016). A Cognitive Semantic Analysis of the Preposition At. Baghdad University.

Aajami, R. F. (2018a). Applying Cognitive Linguistics to Enhance the Semantics of English at. International Journal of English Linguistics, 8(6). https://doi.org/10.5539/ijel.v8n6p185

Aajami, R. F. (2018b). Cognitive Comprehension of "Beyond \& Behind": An Experimental Study. International Journal of English Linguistics, 8(6). 303-314. https://doi.org/10.5539/ijel.v8n6p303.

Bores, F. (1996). Spatial prepositions and metaphores: a cognitive semantic journy along the up down and thefront back dimensions. Language in Performance, 12.

Bores, F., \& Demecheleer, M. (1998, July). A cognitive semantic approach to teaching prepositions. ELT Journal, 52(3).

Brala, M. (2008). Understanding the English preposition 'at'. Semantics and translation from a cognitive perspective. University of Rijeka, Croatia

Celce-Murica, M., \& Larsen-Freeman, D. (1999). The Grammar Book: An ESL/ EFL Teachers' Course. Rowely, Mass: New bury Hose

Evans, V. (2012). Cogniotive Linguistics. Brighton: University of Sussex.

Geeraerts, D., \& Cuyckens, H. (2010). Introducing Cognitive Linguistics. Oxford: Oxford Handbook.https://doi.org/10.1093/oxfordhb/9780199738632.013.0001

Khan, S., \& Ali, R. (2017). Acognitive-Semantic Study of the Spatial Preposition (fi) in the Quran. Penerbit Universiti Sains Malaysia. Kemanuslaan The Asian Journal of Humanities, Vol. 24, No.2, p.89-122. https://doi.org/10.21315/kajh2017.24.2.4.

Mohammed,T. M. Q., Abdullah, H.I., \& Hua,T.K. (2012). The Preposition (fii) in the Hoizontaland Vetical Axes as Used in the Taizzi Dialect: A Cognitive Approach. Asian Social Science, Vol.8, No.4. doi:10.5539/ass.v8n4p99.

Mueller, C. (2016). A Semantic Account of The English Preposition FOR Based on a Cognitive Linguistics Framework. The Bulletin of the Faculty of Humanities, 53, 124.

Navarro, I. (2000). Acognitive-Semantic Analysis of the English Lexical Unit in. C.I.F www.Researchegate.net/puplication/28079614, pp.. 189- 220. Doi: 18172/cif.2227.

Saed, A. H., \& Yassin, B. (2017). Jordanian Undergraduate Students' Use of English Prepositional Verbs: Analytical Study. Canadian Social Science, 13(4), 68-75.

Suzanne, N. (2017). An Analysis of Preposition Errors: The Case in, on, and at. Universitas Negeri Padang. Lingua Didaktika, Vol.11, No.1, p.13-21. Doi: 10.24036/id.v11i1.7408.

Tyler, A., \& Evans, V. (2003). The Semantics of English Prepositions: spatial scenes, embodied meaning, and cognition. Cambridge: UK Cambridge University Press. https://doi.org/10.1017/CBO9780511486517

Tyler, A., \& Evans, V. ( 2004). Applying Cognitive Linguistics to Pedagogical Grammar: The case of Over. In M. Achard \& S. Niemeier (Eds.), Cognitive Linguistics, Second Language Aquisition, and Foreign language Teaching (pp. 257-280). Berlin: Mont de Gruyter. https://doi.org/10.1515/9783110199857.257

Tyler, A., Mueller, C. M., \& Ho, V. (2011). Applying Cognitive Linguistics to Learning the English Prepositions To, For, and At: an experimental investigation. Vigo International Journal of Applied Linguistics, (8), 122-140.

Ungerer, F., \& Schmid, H. (2001). An Introduction to Cognitive Stylistics. Beijing: Foreign Language Teaching and Research press. 